\title{
HbA1c analysis by Capillary electrophoresis - comparison with chromatography and an immunological method
}

Olav Klingenberg ${ }^{1,2}$, Torill Furuset ${ }^{1}$, Camilla R. Hestbråten ${ }^{1}$, Marit H. Hallberg ${ }^{1}$, Astrid Steiro $^{1}$, Iren R. Orset ${ }^{1}$ and Jens P. Berg ${ }^{1,2}$

1.Department of Medical Biochemistry, Oslo University Hospital, Oslo, Norway

2. Institute of Clinical Medicine, University of Oslo, Oslo, Norway

Corresponding Author: Olav Klingenberg, Department of Medical Biochemistry, Oslo University Hospital, PO box 4956 Nydalen, 0424 Oslo, Norway

E-mail: oklingen@ ous-hf.no Tel.: +47 22119468

Running title: HbA1c by Capillarys II-CP

E-mails of authors:

oklingen@ous-hf.no, camhes@ous-hf.no, mhallber@ous-hf.no, asteiro@ ous-hf.no, uxorir@ous-hf.no, j.p.p.berg@medisin.uio.no 


\begin{abstract}
Background.

Haemoglobin A1c (HbA1c) has become an even more important analyte for clinical laboratories during recent years with the introduction of its diagnostic use for diabetes mellitus. Several different analytical principles can be used, each with their advantages and disadvantages.
\end{abstract}

Aim.

We wanted to compare Sebia Capillarys 2 Flex Piercing (Capillarys) with our routine HbA1c methods, which were an HPLC method (Tosoh G7) and an immunoassay (Tina-Quant on Roche Modular P) by analysing a large clinical material. Furthermore, we investigated sample stability.

Methods.

HbA1c analysis was performed in parallel by all three methods for more than 600 patient samples including common and some rare haemoglobin variants, as well as for several controls, some with set target values. Sample stability at room temperature and refrigerated was assessed for up to seven days.

Results.

Capillarys produced generally somewhat lower HbA1c values than both comparison methods, apparently due to positive bias for the comparison methods. Leaving out samples with haemoglobin variants, we found a mean bias $(95 \% \mathrm{CI})$ for Capillarys compared to Tosoh G7 (without factorization) and Modular of -0.39 (-0.40 to -0.38$)$ and $-0.16(-0.17$ to -0.14$) \%$ HbA1c, respectively. HbA1c results were similar between instruments for samples from dialysis patients as well as for samples with heterozygous common haemoglobin variants, except that Tosoh G7 reported too low results in the presence of $\mathrm{Hb}$ E. For heterozygous $\mathrm{Hb}$ Raleigh, Capillarys and the immunoassay gave similar results.

Conclusion.

Capillarys is a convenient instrument for routine $\mathrm{HbA} 1 \mathrm{c}$ analysis.

Key words: HbA1c, Haemoglobin variant, Capillary electrophoresis, Hb Raleigh, Hb Lepore 


\section{Introduction}

Haemoglobin A1c (HbA1c) is the fraction of haemoglobin with a glycation modification of the amino terminal valine residue of the $\beta$-globin chain [1]. The HbA1c level closely correlates with average plasma glucose the preceding 2-3 months [2] and has been used for monitoring of diabetes control for three decades [1]. In 2011 the World Health Organisation accepted $\mathrm{HbA} 1 \mathrm{c}$ also for diagnostic use for diabetes mellitus with a cut-off at $\geq 6.5 \%$ [3]. Methods with several different principles for HbA1c analysis are in clinical use, such as ion exchange chromatography, boronate affinity chromatography and immunoassays [1] as well as enzymatic methods and capillary electrophoresis [4].

The Sebia Capillarys 2 Flex Piercing (Capillarys) system for HbA1c analysis based on capillary electrophoresis has been available for a few years [5]. Several evaluations of the instrument have been published[5-8] demonstrating its usefulness for clinical routine. We wanted to compare the Capillarys instrument with the two methods we used in our clinical routine in a large clinical material of more than 600 samples including samples from dialysis patients and from patients with haemoglobin variants. The comparison methods were cation exchange chromatography (Tosoh G7) and immunoassay (Roche Tina-Quant HbA1c Gen. 3). Reagent cost per test was similar between Tosoh G7 and Capillarys, while reagents for the immunoassay were approximately twice the price. In addition, since the vendor's specification of sample stability at room temperature extends to only $18 \mathrm{~h}$ for Capillarys[9], we tested sample stability for up to one week at both room temperature and refrigerated. 


\section{Materials \& Methods}

\section{Samples and controls}

For method comparison, a total of 658 samples were included during the period January to June 2012. Due to missing data from at least one instrument, 34 samples were excluded from further analysis, leaving 624 patient samples with full datasets available for analysis. Most samples (604 of samples with full datasets) were referred for HbA1c analysis, among which 10 samples were from haemodialysis patients. In addition, 20 samples referred for haemoglobinopathy testing were included. Venous samples with di-potassium ethylenediaminetetraacetic acid as anticoagulant were used.

In the beginning of the study period, all samples referred for Hbalc analysis were included. Later, to obtain a more uniform distribution within the measuring range, samples with low or high $\mathrm{HbA1c}$ results were selected. Samples were analysed at the day of sampling on Roche Modular as part of our clinical laboratory routine and then stored overnight at $4{ }^{\circ} \mathrm{C}$. Next day, samples were first analysed on the Capillarys Cap Piercing and then on Tosoh G7 within the same day.

A high control made from pooled patient samples and a normal control donated by a healthy volunteer were analysed once daily on Roche Modular $(n=23)$, twice daily on Tosoh G7 $(\mathrm{n}=44)$ and once daily on all available capillaries on Sebia Capillarys 2 Cap Piercing (CP) $(\mathrm{n}=189)$. Both controls were stored in aliquots at $-70{ }^{\circ} \mathrm{C}$ to avoid freeze-thaw cycles. They were analysed the same day as they were thawed.

Two external controls from the Norwegian Clinical Chemistry EQA Program with reference method assigned values from European Reference Laboratory were also analysed (only once on each instrument due to sample volume limitations).

For stability experiments, 24 clinical routine samples without haemoglobin variants were selected and analysed on the Capillarys. Of these, 20 samples were analysed once daily Monday to Friday and again the following Monday. Four samples were analysed once daily Tuesday to Friday and again the following Monday. Storage at both room temperature (20$\left.25^{\circ} \mathrm{C}\right)$ and in refrigerator $\left(4^{\circ} \mathrm{C}\right)$ was investigated. To control for minor day-to-day variation, results were normalized to the mean of two instrument controls run on all capillaries each day. All samples were anonymized before inclusion and the study was approved by the Hospital Information Security Unit.

\section{Methods}

For method comparison, HbA1c was analysed on three instruments: i) On the Roche Modular P instrument (Roche, Switzerland), the Tina-Quant HbA1c Gen. 3 method based on a competitive turbidimetric inhibition immunoassay in hemolysate with Roche C.f.a.s. HbA1c as calibrator was used (hereafter denoted as Modular). ii) The Tosoh G7 HPLC instrument (Tosoh Corporation, Japan) was used in the Variant mode (2.2 min program) with Tosoh calibrator (hereafter denoted as Tosoh G7). Both instruments were used for routine HbA1c analysis at our department and were following external quality assurance programs. The Sebia Capillarys 2 Flex Piercing instrument (Sebia, France) (hereafter denoted as Capillarys) was kindly provided for our disposition from the national Sebia distributor for the method comparison in 2012. For the sample stability experiments in 2015 it was a routine HbA1c instrument. The Hba1c-program was used with HbA1c Capillarys calibrators (References 4755).

All methods were run in accordance with the respective companies' instructions and all instruments reported $\mathrm{HbA1c}$ with \% as unit as is still the standard in Norway.

Serum creatinine concentration was measured on Roche Modular P using an enzymatic method (CREA plus). Hemoglobinopathies were analyzed by cation exchange 
chromatography on BioRad Variant (Hercules, CA) with Beta-thal short program and/or by capillary electrophoresis on Sebia Capillarys II by Sebia Capillarys Hemoglobin assay. Haemoglobin variants were analysed on both instruments. In the case of Hb Raleigh, betaglobin gene sequencing was also performed.

\section{Statistical evaluation}

Methods were compared by Deming regression, Bland-Altman plots and related samples Ttest. Sample stability was assessed by Kruskal-Wallis test since Shapiro-Wilk test for normality failed. Dunn's method was used to identify the day from which a significant change occurred. SigmaPlot, Minitab 17 and SPSS version 21 were used for statistical comparisons.

\section{Results}

\section{Method comparison - Controls}

The high and normal level controls were both analysed once daily (Modular), twice daily (Tosoh G7) or once daily on each capillary (Capillarys) during the study period. Tosoh G7 and Capillarys showed similar precision, with CV well below the specification of less than $2 \% \mathrm{CV}$ for diagnostic use[10], while Modular presented poorer precision (Fig 1A, Table I). Two samples from the Norwegian Clinical Chemistry EQA Program with assigned values from European Reference Laboratory (ERL) were also analysed (samples 2012-02 and 201203). All three studied methods gave higher results than the ERL assigned values (Fig. 1B), but Capillarys was closer to the assigned values than were the other two instruments.

During the study sampling period in 2012, the Norwegian Clinical Chemistry EQA Program recommended Norwegian users of Tosoh G7 and G8 to factorize HbA1c-results due to systematically high results for the instrument group in their external quality control program [11]. When we included the recommended factor (0.955), the Tosoh G7-results for the ERL controls were similar to the Capillarys results (not shown).

\section{Method comparison - Patient samples}

Complete datasets from all three instruments were available for 624 samples. Distribution of results is shown in Fig 2 as measured by Capillarys, displaying good coverage for the diagnostic cut-off for diabetes mellitus at $6.5 \% \mathrm{HbA} 1 \mathrm{c}$ [3].

In accordance with results for the controls, there were systematic differences between the instruments. Leaving out samples with haemoglobin variants, we found a mean bias $(95 \% \mathrm{CI})$ for Capillarys compared to Tosoh G7 (without factorization) and Modular of -0.39 (-0.40 to $0.38)$ and -0.16 (-0.17 to -0.14$) \% \mathrm{HbA} 1 \mathrm{c}$, respectively. In fig 3 are shown Deming regression and Bland-Altman plots for the comparisons. Samples with haemoglobin variants are excluded in fig. 3. One analytical outlier on Modular is displayed well below the regression lines in fig $3 \mathrm{C}$ and $\mathrm{E}$. The result for this sample from Modular was $6.9 \% \mathrm{HbA} 1 \mathrm{c}$, while Capillarys and Tosoh G7 results were $5.0 \%$ and $5.2 \%$, respectively.

Samples with $\mathrm{HbA} 1 \mathrm{c}$ requests from ten dialysis patients with creatinine concentrations ranging from 430 to $700 \mu \mathrm{mol} / \mathrm{L}$ were also included. For these samples, only similar instrument differences as noted in the general comparison were observed.

\section{Hemoglobin variants}

We included samples with common heterozygous haemoglobin variants, eleven $\mathrm{Hb} \mathrm{E}$, two $\mathrm{Hb} \mathrm{S}$, one $\mathrm{Hb} \mathrm{C}$ and one $\mathrm{Hb}$ D-Punjab. HbA1c results from Modular and Capillarys were similar for all haemoglobin variants, while results for heterozygous $\mathrm{Hb} \mathrm{E}$ samples were clearly lower from Tosoh G7 than from the two other instruments (Fig. 4A). Also samples with more rare haemoglobin variants were analysed. Heterozygous Hb Raleigh 
was not separated from $\mathrm{HbA}$ in the Capillarys electropherogram (Fig. 4B) while the variant eluted with HbA1c on Tosoh G7 (Fig. 4C) and the HbA1c result could thus not be reported from this instrument. $\mathrm{HbA1c}$ results from Capillarys and Modular for the sample with $\mathrm{Hb}$ Raleigh were 4.1 and $4.2 \%$ respectively.

Heterozygous $\mathrm{Hb}$ Lepore gave similar HbA1c results from all three instruments (5.1-5.4 \%). $\mathrm{Hb}$ Lepore migrated as a sharp peak between $\mathrm{HbF}$ and $\mathrm{HbA} 2$ in the Capillarys electropherogram (Fig. 4 D) while it generated a number-of-peaks flag on Tosoh G7 (Fig. 4E). As expected, for several samples lacking the normal $\mathrm{HbA}$ (homozygous $\mathrm{Hb} \mathrm{S}, \mathrm{HbD} / \beta$ thalassemia, $\mathrm{Hb} \mathrm{E} / \beta$-thalassemia) no result for $\mathrm{HbA} 1 \mathrm{c}$ was reported from Capillarys.

\section{Sample stability}

Referred clinical samples with HbA1c concentrations from $4.6 \%$ to $11.4 \%$ were included. Storage for up to three $(n=24)$ and four $(n=20)$ days at room temperature did not result in a significant change in $\mathrm{HbA1c}$ concentration $(\mathrm{p}=0.12$, Kruskal-Wallis test). Storage for 6-7 days, however, reduced HbA1c concentration ( $\mathrm{p}<0.05$, Dunn's test), Fig. 5A. Storage at $4{ }^{\circ} \mathrm{C}$ for up to one week did not result in any significant change in HbA1c concentration $(p=0.57$, Kruskal-Wallis test, $\mathrm{n}=12$ ), Fig. 5B.

\section{User friendliness}

The laboratory technologists found the Capillarys instrument easy to operate. The instrument had capacity for analysing 35-40 samples/h and walk-away capacity for 108 samples with cap piercing functionality. We found the mosaic screen displaying 48 colour coded results to give a good overview. We experienced one episode of clogging of one of the capillaries during the study period in 2012, but this problem was solved by rinsing with concentrated hypochlorite. No capillary clogging has occurred later.

The Capillarys electropherogram provided good separation of $\mathrm{HbA}, \mathrm{HbA} 1 \mathrm{c}$ and $\mathrm{HbA} 2$ as well as of common haemoglobin variants and was considered easier to interpret than the more complicated chromatogram from Tosoh G7.

\section{Discussion}

The present study confirmed that Capillarys is a suitable instrument for HbA1c analysis in clinical routine with sufficiently good precision to be used diagnostically[10]. It also provides data to extend sample stability at room temperature beyond the eighteen hours as stated by Sebia [9].

At the time we performed the method comparison, it became evident that Tosoh G7 produced slightly too high results, as the here presented comparisons with Capillarys also suggest. As mentioned above, the Norwegian Clinical Chemistry EQA Program advised to factorize HbA1c results from Tosoh G7 and G8 by multiplying by the factor 0.955 [11]. The second comparison method, Tina-Quant HbA1c Gen 3 on the Roche Modular P instrument, was by the end of 2014 phased out from the Norwegian market by Roche due to poor precision. The data here provided show that the method also had a positive bias.

Sufficiently long sample stability at ambient temperature is important when samples are to be sent by post. Sebia states that samples can be stored for 18 hours maximum at room temperature [9], which is clearly too short for mailed samples. We found a significant reduction in HbA1c concentration after 6-7 days, but samples were stable for up to four days of storage at room temperature, which is sufficient for most practical purposes. In the sample stability experiment, the same set of samples was used for the room temperature and refrigerator studies. Due to scarce volume in several cases, only twelve samples could be 
assayed at both temperatures. Since Sebia states that refrigerated samples can be analysed for up to seven days after sampling [9], we prioritized samples for the room temperature part of the study to possibly extend the very limited room temperature stability advised by the vendor.

Haemoglobin variants present as important potential interferences in $\mathrm{HbA} 1 \mathrm{c}$ analysis. The four common variants $\mathrm{Hb} \mathrm{S}, \mathrm{Hb} \mathrm{C}, \mathrm{Hb} \mathrm{D}-\mathrm{Punjab}$ and $\mathrm{Hb} \mathrm{E}$ in the heterozygous states do not interfere with Capillarys HbA1c measurements [9;12]. Underestimation of HbA1c in the presence of $\mathrm{Hb} \mathrm{E}$ with Tosoh G7, as shown in the present work, is well documented[12]. However, since more than 1250 haemoglobin variants have been reported[13] this is a large field to map. Hb Raleigh is a rare variant in which the first amino acid in the mature betaglobin is changed from valine to alanine, and this alanine is posttranslationally modified by acetylation of the $\alpha$-amino group $[14 ; 15]$. The acetylation renders the amino-terminus of the $\beta$-globin chain resistant to glycation $[14 ; 16]$ but also impacts charge in a manner to make $\mathrm{Hb}$ Raleigh mimic HbA1c by cation exchange HPLC[14]. Thus, HbA1c can not be used as a representative measure of average glucose concentration in the presence of $\mathrm{Hb}$ Raleigh. Unfortunately, due to anonymization of samples in the present study, we were not able to compare $\mathrm{HbA} 1 \mathrm{c}$ with any glucose measurements in the patient with $\mathrm{Hb}$ Raleigh. We found that Capillarys and Modular (immunoassay) gave similar HbAlc values for this sample, which is in line with the findings that the variant was not separated from HbA by capillary electrophoresis and apparently did not directly interfere with the HbA1c peak. Thus, the reported $\mathrm{HbA1c}$ values from Capillarys and Modular in the presence of $\mathrm{Hb}$ Raleigh may represent a correct measure of glycated amino-terminus of $\beta$-globin as a fraction of total $\beta$ globin (Raleigh + wild-type), but this value underestimates average glucose concentration.

In conclusion, Capillarys is a convenient instrument for $\mathrm{HbA} 1 \mathrm{c}$ analysis with good separation of peaks, making it easy to interpret the electropherogram in most cases. In some cases, as for $\mathrm{Hb}$ Raleigh, interpretation is difficult whatever instrument is used for analysis.

\section{Acknowledgement}

We are grateful to the Norwegian Clinical Chemistry EQA Program for letting us use their controls with assigned values. 
Table I. Imprecision as evaluated by analysis of controls at two levels.

\begin{tabular}{|l|l|c|c|}
\hline Control & Instrument & Mean (HbA1c \%) & CV (\%) \\
\hline \multirow{3}{*}{ High } & Roche Modular P & 9.96 & 1.62 \\
\cline { 2 - 4 } & Tosoh G7 & 10.01 & 1.28 \\
\cline { 2 - 4 } & Sebia Capillarys 2 FP & 9.47 & 0.91 \\
\hline \multirow{3}{*}{ Low } & Roche Modular P & 6.00 & 2.10 \\
\cline { 2 - 4 } & Tosoh G7 & 6.06 & 0.80 \\
\cline { 2 - 4 } & Sebia Capillarys 2 FP & 5.74 & 1.32 \\
\hline
\end{tabular}


Figure legends

Figure 1. Instrument comparisons using controls. A; Results from analysis of high and low controls are plotted as mean and SD. B; Results from analysis of two controls from the Norwegian Clinical Chemistry EQA Program with assigned European Reference Laboratory values. Tosoh G7 results were not factorized.

Figure 2. Distribution of HbA1c results of included samples as analysed by Capillarys.

Figure 3. Deming regression and Bland-Altman plots for method comparisons. In the BlandAltman plots are shown mean bias and 95\% limits of agreement. Samples with haemoglobin variants were excluded. Tosoh G7 results were not factorized.

Figure 4. Haemoglobin variants. Panel A; Results of HbA1c measurements for samples with heterozygous common haemoglobin variants superimposed on the Deming regression from fig. $3 \mathrm{~A}$. Tosoh $\mathrm{G} 7$ results were not factorized. The variants are represented by letters; $\mathrm{E}=\mathrm{Hb}$ E/A, S=Hb S/A, D=Hb D-Punjab/A, C=Hb C/A.

Panels B-E; Capillarys electropherogram (B,D) and Tosoh $\mathrm{G} 7$ chromatogram $(\mathrm{C}, \mathrm{E})$ for heterozygous $\mathrm{Hb}$ Raleigh $(\mathrm{B}, \mathrm{C})$ and $\mathrm{Hb}$ Lepore $(\mathrm{D}, \mathrm{E})$.

Figure 5. Sample stability at room temperature $(n=24)(A)$ and refrigerated $(n=12)(B)$ analysed by Capillarys. Percentage of measured value at the day of sampling (day 0 ) is presented as means with $95 \% \mathrm{CI}$.

\section{Reference List}

[1] Miedema K. Standardization of HbA1c and Optimal Range of Monitoring. Scand J Clin Lab Invest 2005;65:61-72.

[2] Nathan DM, Kuenen J, Borg R, Zheng H, Schoenfeld D, Heine RJ. Translating the A1C assay into estimated average glucose values. Diabetes Care 2008;31:1473-8.

[3] Use of glycated haemoglobin (HbA1c) in the diagnosis of diabetes mellitus Abbreviated report of a WHO consultation. Available at: http://www.who.int/diabetes/publications/diagnosis_diabetes2011/en/ (accessed Dec. 2016)

[4] List of NGSP Certified Methods. Available at: http://www.ngsp.org/certified.asp (accessed Dec. 2016

[5] Jaisson S, Leroy N, Meurice J, Guillard E, Gillery P. First evaluation of Capillarys 2 Flex Piercing(R) (Sebia) as a new analyzer for HbA1c assay by capillary electrophoresis. Clin Chem Lab Med 2012;50:1769-75.

[6] Weykamp C, Waenink-Wieggers H, Kemna E, Siebelder C. HbA1c: performance of the Sebia Capillarys 2 Flex Piercing. Clin Chem Lab Med 2013;51:e129-e131.

[7] Marinova M, Altinier S, Caldini A, Passerini G, Pizzagalli G, Brogi M, Zaninotto M, Ceriotti F, Plebani M. Multicenter evaluation of hemoglobin A1c assay on capillary electrophoresis. Clin Chim Acta 2013;424:207-11. 
[8] Rollborn N, Akerfeldt T, Nordin G, Xu XY, Mandic-Havelka A, Hansson LO, Larsson A. Analysis of HbA1c on an automated multicapillary zone electrophoresis system. Scand J Clin Lab Invest 2017;77:15-8.

[9] Sebia. CAPILLARYS Hb A1c USING THE CAPILLARYS 2 FLEX-PIERCING INSTRUMENT. Available at: http://www.ilexmedical.com/files/Sebia\%20inserts/CAPILLARYS_Hb_A1c.pdf (accessed 29.12.2016).

[10] Little RR, Rohlfing CL, Sacks DB. Status of hemoglobin A1c measurement and goals for improvement: from chaos to order for improving diabetes care. Clin Chem 2011;57:205-14.

[11] Letter from NKK (Norwegian Clinical Chemistry EQA Program) 29th of February, 2012. 2012.

[12] NGSP. HbA1c Assay Interferences . Available at: http://www.ngsp.org/interf.asp (accessed Dec. 2016)

[13] HbVar: A Database of Human Hemoglobin Variants and Thalassemias. Available at: http://globin.bx.psu.edu/hbvar/menu.html (accessed Dec. 2016)

[14] Chen D, Crimmins DL, Hsu FF, Lindberg FP, Scott MG. Hemoglobin Raleigh as the cause of a falsely increased hemoglobin A1C in an automated ion-exchange HPLC method. Clin Chem 1998;44:1296-301.

[15] Moo-Penn WF, Bechtel KC, Schmidt RM, Johnson MH, Jue DL, Schmidt DE, Jr., Dunlap WM, Opella SJ, Bonaventura J, Bonaventura C. Hemoglobin Raleigh (beta1 valine replaced by acetylalanine). Structural and functional characterization. Biochemistry 1977;16:4872-9.

[16] Jain N, Kesimer M, Hoyer JD, Calikoglu AS. Hemoglobin Raleigh results in factitiously low hemoglobin A1c when evaluated via immunoassay analyzer. J Diabetes Complications 2011;25:14-8. 
A

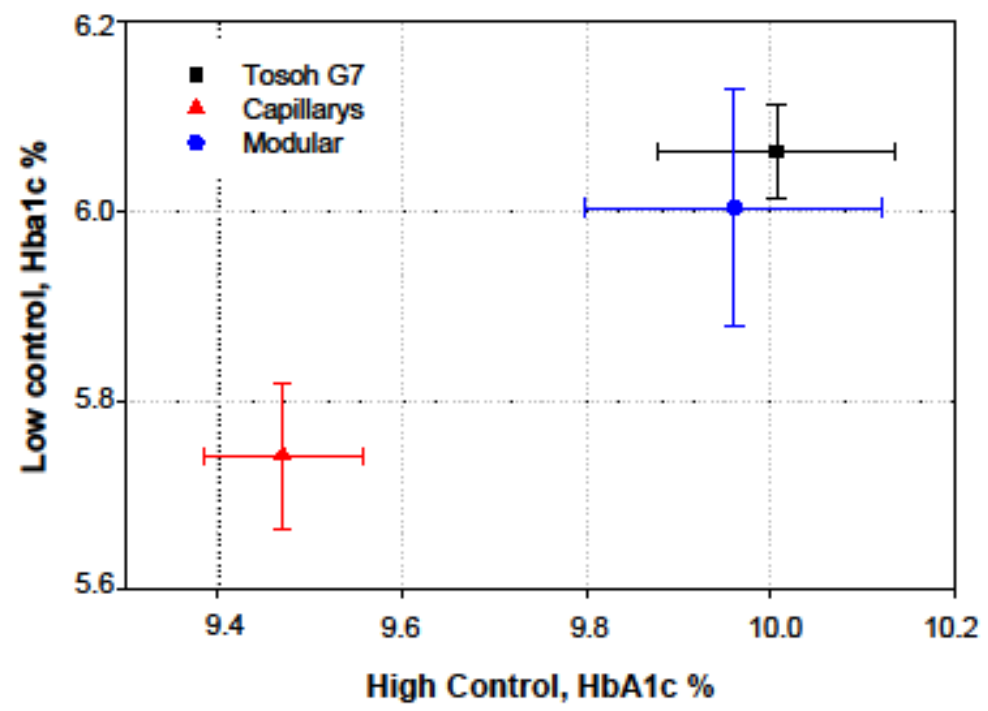

B

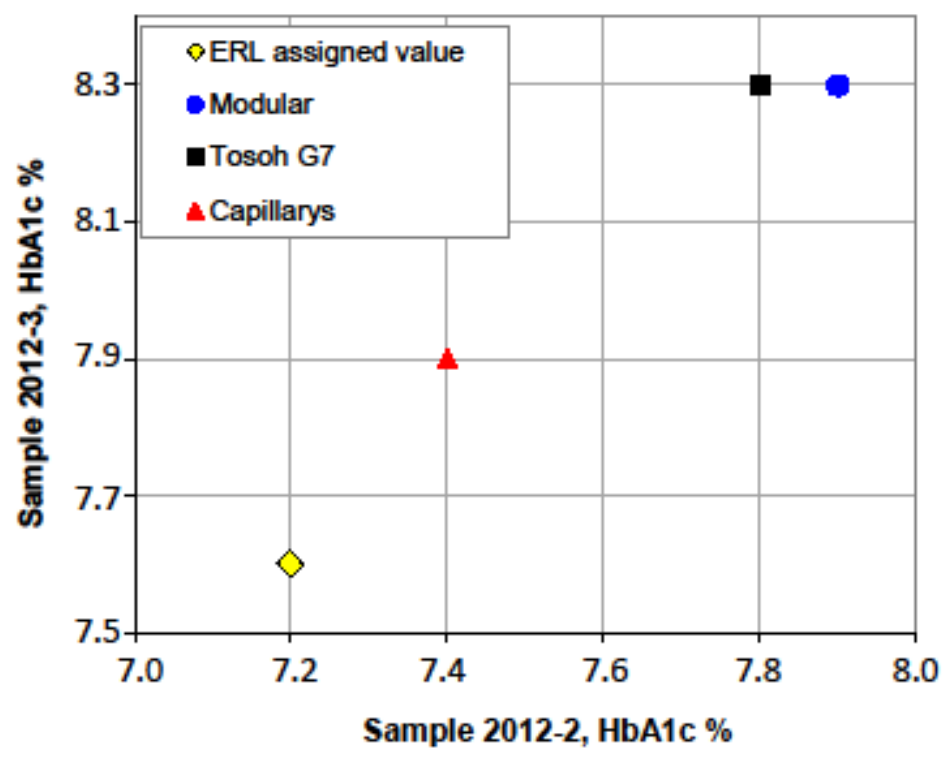

Figure 1. Instrument comparisons using controls. A; Results from analysis of high and low controls are plotted as mean and SD. B; Results from analysis of two controls from the Norwegian Clinical Chemistry EQA Program with assigned European Reference Laboratory values. Tosoh G7 results were not factorized. 


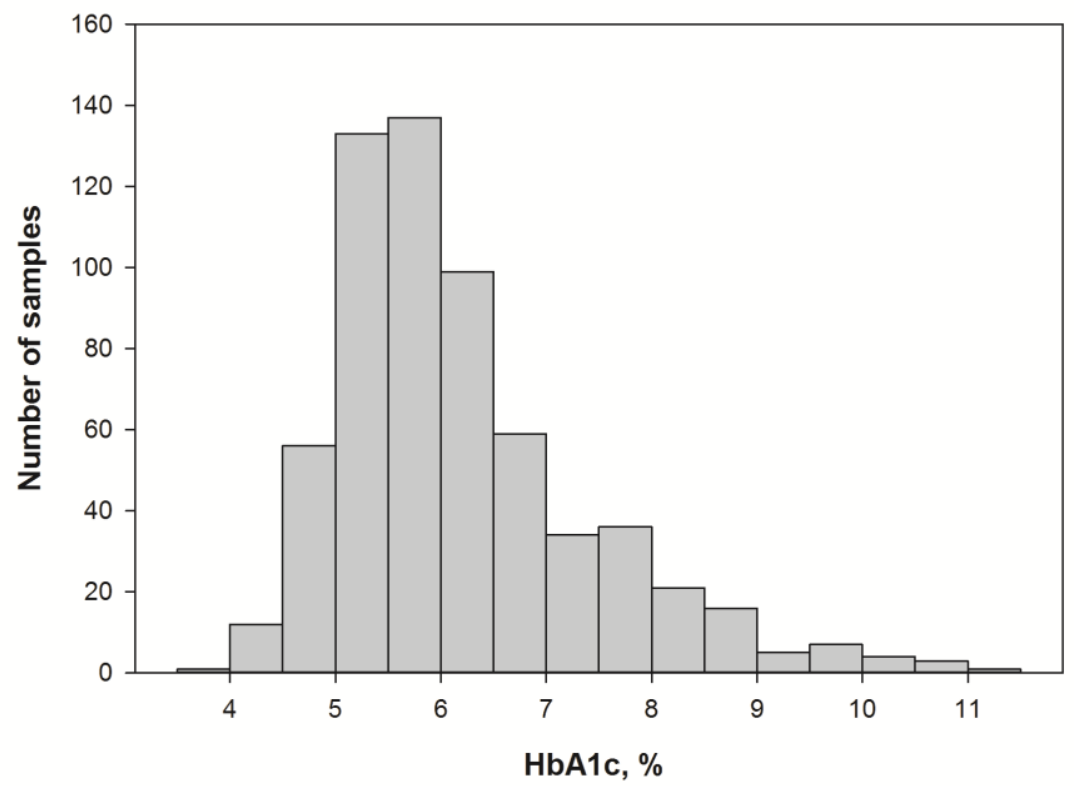

Figure 2. Distribution of HbA1c results of included samples as analysed by Capillarys. 
A

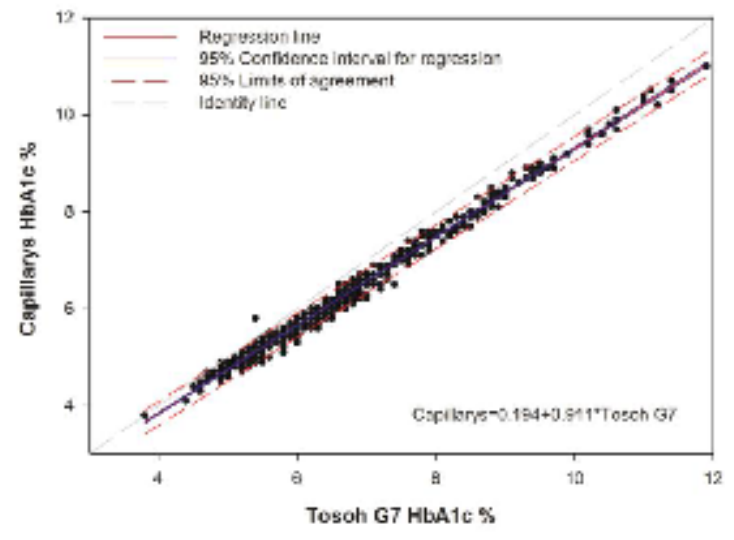

C

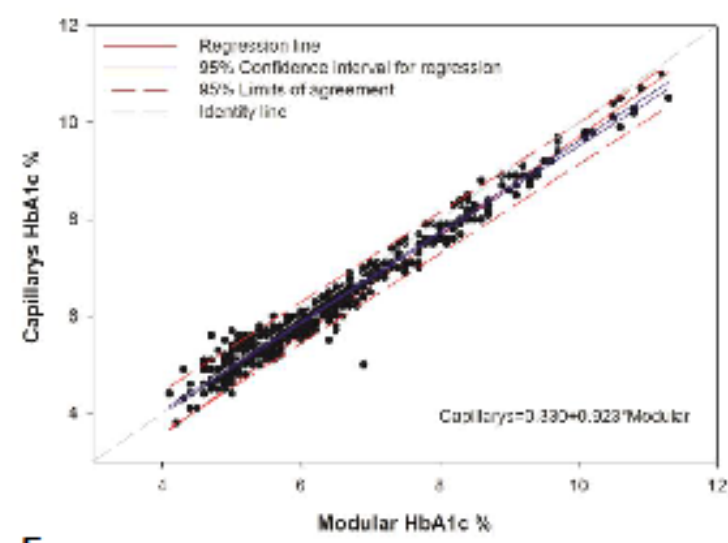

$\mathrm{E}$

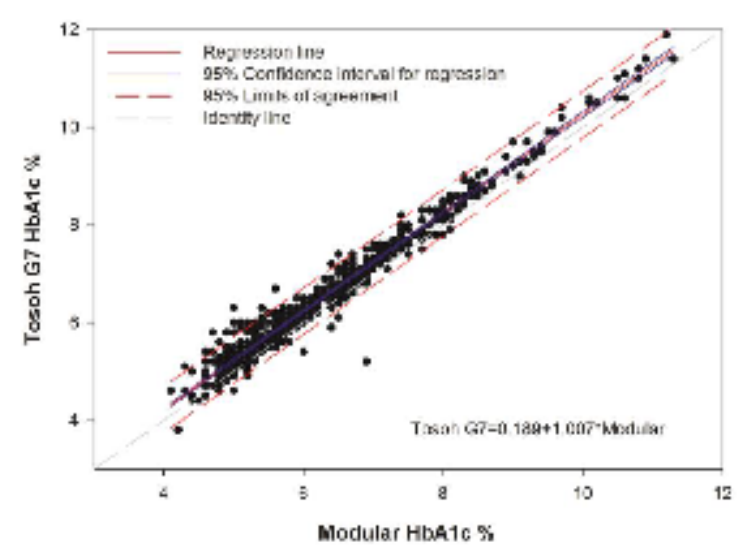

B

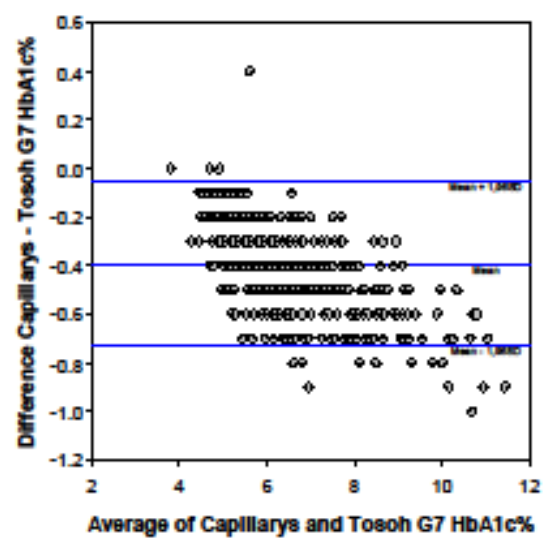

D

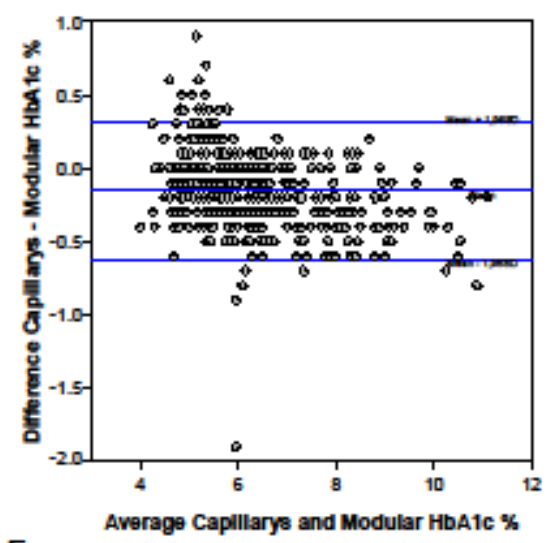

$\mathrm{F}$

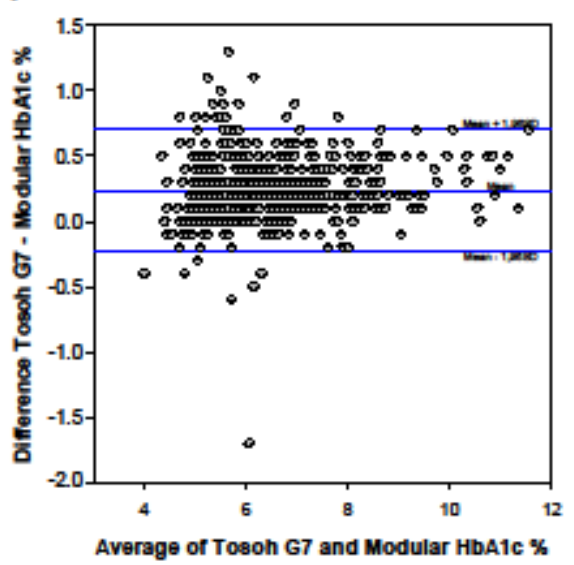

Figure 3. Deming regression and Bland-Altman plots for method comparisons. In the BlandAltman plots are shown mean bias and 95\% limits of agreement. Samples with haemoglobin variants were excluded. Tosoh G7 results were not factorized. 

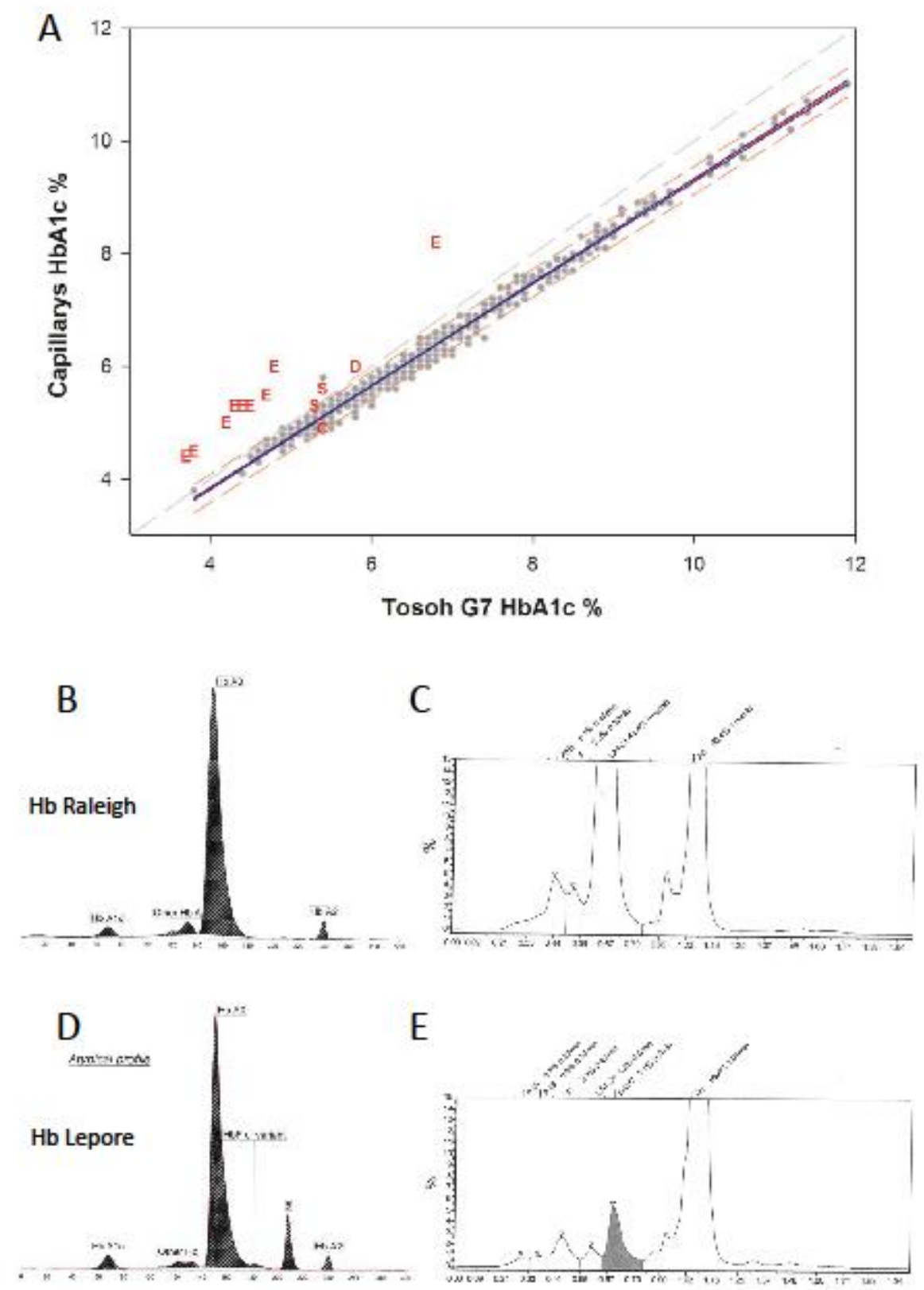

Figure 4. Haemoglobin variants. Panel A; Results of HbA1c measurements for samples with heterozygous common haemoglobin variants superimposed on the Deming regression from fig. 3 A. Tosoh $\mathrm{G} 7$ results were not factorized. The variants are represented by letters; $\mathrm{E}=\mathrm{Hb}$ E/A, S=Hb S/A, D=Hb D-Punjab/A, C=Hb C/A.

Panels B-E; Capillarys electropherogram (B,D) and Tosoh $G 7$ chromatogram $(C, E)$ for heterozygous $\mathrm{Hb}$ Raleigh $(\mathrm{B}, \mathrm{C})$ and $\mathrm{Hb}$ Lepore $(\mathrm{D}, \mathrm{E})$. 
A

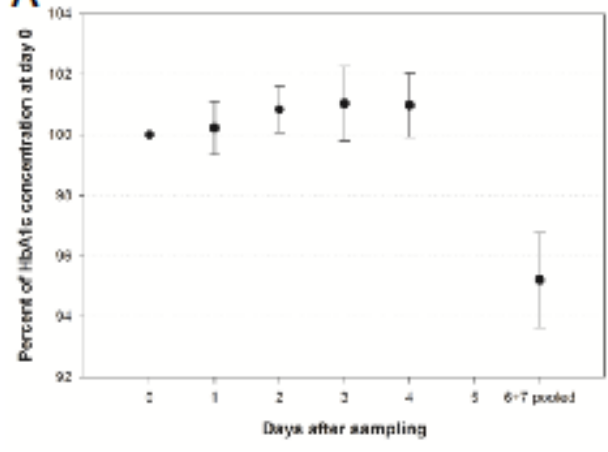

B

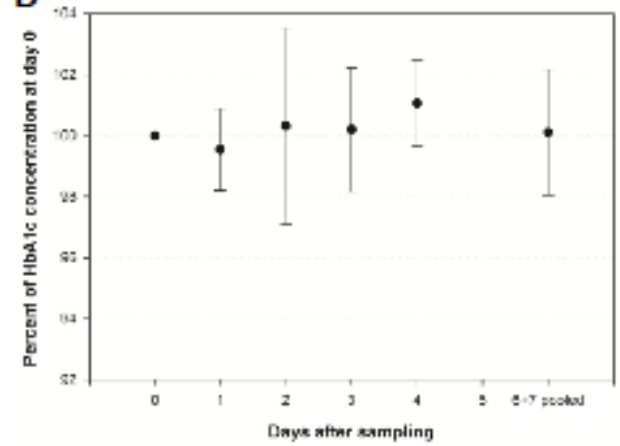

Figure 5. Sample stability at room temperature $(n=24)(A)$ and refrigerated $(n=12)(B)$ analysed by Capillarys. Percentage of measured value at the day of sampling (day 0 ) is presented as means with $95 \% \mathrm{CI}$. 